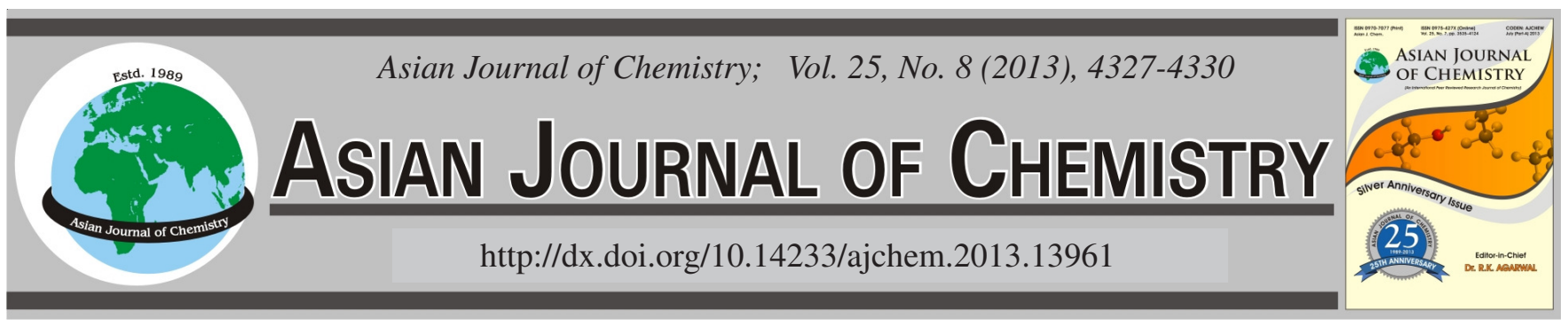

\title{
Design, Synthesis and Characterization of Novel Biodegradable Poly(urethane-urea) for Biomedical Applications
}

\author{
N. $\mathrm{Hu}^{1}$, C.S. RuAN ${ }^{2, *}$, X.Q. NING ${ }^{1}$ and C.M. Xu ${ }^{1}$
}

${ }^{1}$ College of Bioengineering, Chongqing University, Chongqing 400030, P.R. China

${ }^{2}$ Center for Human Tissue and Organs Degeneration, Institute of Biomedicine and Biotechnology, Shenzhen Institutes of Advanced Technology, Chinese Academy of Sciences, Shenzhen 518055, P.R. China

*Corresponding author: E-mail: henry-cs@ hotmail.com

(Received: 25 April 2012;

Accepted: 9 February 2013)

AJC-12956

\begin{abstract}
A series of novel biodegradable poly(urethane-urea) were designed and synthesized based on poly(D,L-lactic acid)diol, 1,6-hexamethylene diisocyanate and piperazine. Their structure and mechanical properties were characterized by fourier transform infrared spectroscopy, nuclear magnetic resonance and dynamic mechanical analysis. These results revealed that the successful introduction of the piperazine into the backbone of the biodegradable poly(urethane-urea) could make them have excellent mechanical properties. Increasing the hard segments of biodegradable poly(urethane-urea), the $\mathrm{T}_{\mathrm{g}}$ and storage modulus of biodegradable poly(urethane-urea) increased from 29.35 to $55.63{ }^{\circ} \mathrm{C}$ and from 2523 to $3295 \mathrm{MPa}$, respectively. Moreover, in vitro degradation tests confirmed that piperazine as the chain extender would improve the biodegradable stability of biodegradable poly(urethane-urea) and regulate its degradation rate.
\end{abstract}

Key Words: Poly(urethane-urea), Poly(D,L-lactic acid)diol, Piperazine, Mechanical property, In vitro degradation. ᄂ - - - - - - - - - - - - - - - - - - - - - - - - - -

\section{INTRODUCTION}

Biodegradable polyurethanes (BPUs) have been attracted great attention for application in tissue engineering ${ }^{1,2}$, orthopaedic $^{3}$ and drug delivery ${ }^{4}$. However, to date, few biodegradable polyurethanes have been developed to satisfy for the practical application in bone tissue repair, the most possible reason was that the desirable biodegradable polyurethanes for bone tissue engineering should host such properties as biocompatibility, biodegradability and appropriate mechanical properties $^{1-3,5-7}$. Soft segment, chain extender and diisocyanate are typical factors responsible for these properties. To meet those needs of biomedical applications, in this work, a novel biodegradable polyurethanes was designed and synthesized based on poly(D, L-lactic acid)diol (PDLLA diol), hexamethylene diisocyanate and piperazine. During the synthesis process, piperazine (PP), a six-member secondary diamine, was twice introduced into the backone of polyurethane. One was that piperazine was selected as the chain extender, the other was that piperazine was employed as a co-initiator of lactide, producing the amorphous poly (D, L-lactic acid) (PDLLA) diol as the soft segment. The diamine instead of diols as the chain extender, which would make the polymer contain a large number of urea group in the hard segment. Since urea groups rather than urethane are produced in the hard segments, the resulting polyurethanes are called biodegradable poly(urethaneurea) (BPUU). Based on this approach, the BPUUs with piperazine as chain extender would have a number of advantages e.g., firstly, a large of urea groups in the chain of BPUUs, may provide more hydrogen bonds and thus more rigid hard segments compared to polyurethanes ${ }^{8}$ and lots of six-atom rigidity piperazine rings in the hard segment, will enhance the mechanical properties of materials. Secondly, the secondary amine, like primary amine $e^{9,10}$, can also regulate the degradation rate and control the acidity. Finally, piperazine is also a popular drug intermediate with good biocompatibility ${ }^{11,12}$, which would not affect the biocompatibility of BPUUs.

\section{EXPERIMENTAL}

D, L-lactide (D, L-LA) was synthesized and recrystallized in our laboratory. Piperazine in analytical grade was purchased from Kelong Chemical (Chengdu, China). 1,6-hexamethylene diisocyanate (HDI) and stannous octoate [ $\mathrm{Sn}(\mathrm{Oct})_{2}, 95 \%$ ] were purchased from Aldrich. Toluene was distilled over freshly powdered calcium hydride under reduced pressure. All chemicals used in the experiment except for specified description, were analytical grade and obtained from the commercial source.

A series of novel BPUUs were prepared as follows (Scheme-I $)^{13}$ : Firstly, the poly(D,L-lactic acid)diol was 
synthesized by ring opening polymerization of $\mathrm{D}$, L-lactide in the presence of $\mathrm{Sn}(\mathrm{Oct})_{2}$ as an initiator and piperazine as a co-initiator at $150^{\circ} \mathrm{C}$ for $24 \mathrm{~h}$ under vacuum. Then, the predetermined amounts of PDLLA diol, HDI, $\mathrm{Sn}(\mathrm{Oct})_{2}$ and anhydrous toluene were added into a $250 \mathrm{~mL}$ three-neck round-bottomed flask with magnetic stirrer. The prepolymer was obtained at $75^{\circ} \mathrm{C}$ for $3 \mathrm{~h}$. Finally, piperazine dissolved in isopropanol, was added into reaction system slowly for chain extension at $30^{\circ} \mathrm{C}$ for $2 \mathrm{~h}$. Molar number of the added piperazine was equal to the difference between the HDI moles and the macrodiol moles. The BPUU was purified through co-precipitation of toluene/ethanol and dried under vacuum at the room temperature for $48 \mathrm{~h}$.

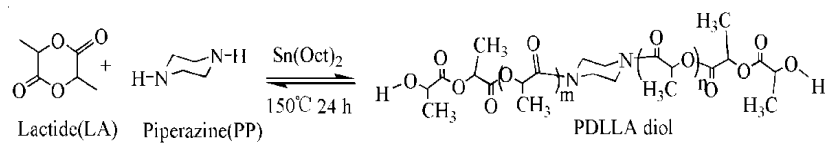

$$
\begin{aligned}
& 0^{\mathrm{C}=}=\mathrm{N}_{\mathrm{N}=\mathrm{C}^{\prime \prime}} \mid \begin{array}{l}
\mathrm{Sn}(\mathrm{Oct})_{2} \\
75^{\circ} \mathrm{C}, 3 \mathrm{~h}
\end{array} \\
& \mathrm{H}-\mathrm{N} \approx \mathrm{N}-\mathrm{H} \mid 30^{\circ} \mathrm{C}, 2 \mathrm{~h}
\end{aligned}
$$

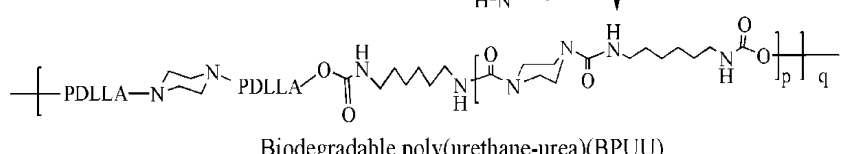

Scheme-I: Synthesis route of biodegradable poly(urethane-urea)

The FTIR spectra of samples were recorded on PerkElimer GX spectrometer (Perkin Elmer, USA) in $\mathrm{KBr}$ discus. Gel permeation chromatography with multi-angle laser light scattering (Wyatt Technology Co., USA) (GPC-MALLS) was used to determine the molecular weight and polydispersity index (PDI) of the BPUUs. Three Agilent 1100 HPLC columns $(300 \times 8.0 \mathrm{~mm})$ were used in series with $\mathrm{THF}$ as the eluent at a flow rate of $1.0 \mathrm{~mL} / \mathrm{min}$ at $25^{\circ} \mathrm{C}$. The number average molecular weight $\left(\mathrm{M}_{\mathrm{n}}\right)$ of the PDLLA diol was determined by ${ }^{1} \mathrm{H}$ NMR. Nuclear magnetic resonance (NMR) was performed on a Bruker AV-4500 spectrometer and the dried samples were dissolved in $\mathrm{CDCl}_{3}$. The mechanical properties and $\mathrm{T}_{\mathrm{g}}$ were determined by dynamic mechanical analysis (DMAQ800, TA Co. USA) (DMA).

\section{In vitro degradation test}

Sample preparation: BPUUs films were prepared according to solution casting method by dissolving BPUUs in 3 wt \% chloroform solution and then casting in glass Petri dishes $(47 \mathrm{~mm})^{14}$. The solvent was allowed to evaporate over $48 \mathrm{~h}$. The formed films were dried in vacuum until a constant weight was achieved. All experiments were performed at room temperature. The obtained glabrous transparent films were cut into $10 \times 10 \mathrm{~mm}$ pieces and UV sterilized for $30 \mathrm{~min}$ before performing degradation study ${ }^{15}$. The samples were placed in $5 \mathrm{~mL}$ vials and assessed in vitro by $\mathrm{pH}$ variation and weight loss rate using double distilled water and PBS solution $(0.1$ $\mathrm{M}, \mathrm{pH}$ 7.4) as degradation medium, respectively. All experiments were performed in a shaking incubator $\left(37 \pm 0.5^{\circ} \mathrm{C}\right.$, $50 \mathrm{rpm})$.

pH value change test during degradation: To detect $\mathrm{pH}$ value of the medium soaked with samples, $5 \mathrm{~mL}$ distilled water was added to the vials. $\mathrm{pH}$ value of the medium was measured once a week for 12 weeks using a pHS-25 pH meter (China). Three samples were prepared for each polymer and the reported $\mathrm{pH}$ was the average of three samples. The change of $\mathrm{pH}$ value of every week was calculated by the difference of $\mathrm{pH}$ value between two weeks.

Weight loss rate: In the weight loss ratio examination, $5 \mathrm{~mL}$ sterile PBS solution was added to the vials and 36 samples were prepared for each polymer. At the end of each week, three vials of each BPUU were taken out, rinsed with distilled water and vacuum dried at room temperature to constant weight. The weight loss ratio and the water absorption ratio were calculated as follows:

$$
\text { Weight } \operatorname{los}(\%)=\frac{\mathrm{W}_{0}-\mathrm{W}_{\mathrm{t}}}{\mathrm{W}_{0}} \times 100 \%
$$

where $\mathrm{W}_{0}$ and $\mathrm{W}_{\mathrm{t}}$ were weights of the sample before and after hydrolytic degradation. The reported weight loss rates were the averages of three samples.

\section{RESULTS AND DISCUSSION}

Characterization by FTIR and NMR: With the PDLLA diol $\left(\mathrm{Mn}=1957\right.$, determined by ${ }^{1} \mathrm{H}$ NMR $)$ as a starting material, a series of BPUUs were prepared. According to the molar ratios of diol/HDI/PP in the reaction feed, BPUUs was divided into four groups, i.e. BPUU2K1.1, BPUU2K1.2, BPUU2K1.3 and BPUU2K1.4, meaning diol/HDI/PP = 1/1.1/0.1, 1/1.2/0.2, $1 / 1.3 / 0.3$ and $1 / 1.4 / 0.4$, respectively. The basic data for these polyurethanes are listed in Table-1. The molecular weight and polydispersity index (PDI) of the polymers were determined with GPC and the $T_{g}$ of BPUUs was determined from the transition of dynamic loss ( $\tan \delta$ ).

TABLE-1

BASIC DATA FOR POLYMERS

\begin{tabular}{lccc}
\hline Polymer & $\mathrm{Mn}\left(10^{4}\right)$ & PDI & $\mathrm{T}_{\mathrm{g}}\left({ }^{\circ} \mathrm{C}\right)$ \\
\hline PDLLA diol & 0.1957 & - & - \\
BPUU2K1.1 & 3.564 & 1.02 & 29.35 \\
BPUU2K1.2 & 4.123 & 1.11 & 38.23 \\
BPUU2K1.3 & 4.766 & 1.19 & 46.29 \\
BPUU2K1.4 & 5.081 & 1.27 & 55.63 \\
\hline
\end{tabular}

Fig. 1 showed FTIR spectra of diol and BPUU2K1.1. Diol (Fig. 1a) exhibited their characteristic peak at $3506 \mathrm{~cm}^{-1}, 1753$ $\mathrm{cm}^{-1}$ and $1657 \mathrm{~cm}^{-1}$, which were attributed to the $\mathrm{OH}$ stretching vibration, the $\mathrm{C}=\mathrm{O}$ stretching vibration and the $\mathrm{N}-\mathrm{C}=\mathrm{O}$ stretching vibration, respectively. The $\mathrm{N}-\mathrm{C}=\mathrm{O}$ stretching vibration revealed that piperazine was successfully inserted into the backbone of PDLLA diol. Compare with Fig. 1a, A peak at $3416 \mathrm{~cm}^{-1}$, due to the $\mathrm{NH}$ stretching vibration instead of the peak at $3506 \mathrm{~cm}^{-1}$, appeared in Fig. 1b. Moreover, typical amide II band at $1524 \mathrm{~cm}^{-1}$ was present and the peak of amide I stretching vibration must coincide with the $\mathrm{N}-\mathrm{C}=\mathrm{O}$ stretching vibration in PDLLA diol, which show in $1657 \mathrm{~cm}^{-1}$. The ${ }^{1} \mathrm{H}$ NMR spectra of PDLLA diol (i) and BPUU2K1.1 (ii) were shown in Fig. 2. In Fig. 2a, Peaks a and b were corresponding to chemical shifts of $-\mathrm{CH}$ and $-\mathrm{CH}_{3}$ of the LA unit and Peaks $a^{\prime}$ and $b^{\prime}$ were corresponding to the chemical shifts of the LA unit in terminal group. The peak c, a symmetrical two-humped peak, was attributed to the proton of piperazine. Comparing 
with the ${ }^{1} \mathrm{H}$ NMR of $\mathrm{D}_{2} \mathrm{~K}$, new characteristic peaks d, e and $\mathrm{f}$ could be easily found, which were assigned to the proton of $-\mathrm{CH}_{2}$ - from HDI. All these spectral changes provided convincing evidence for the formation of polyurethane.

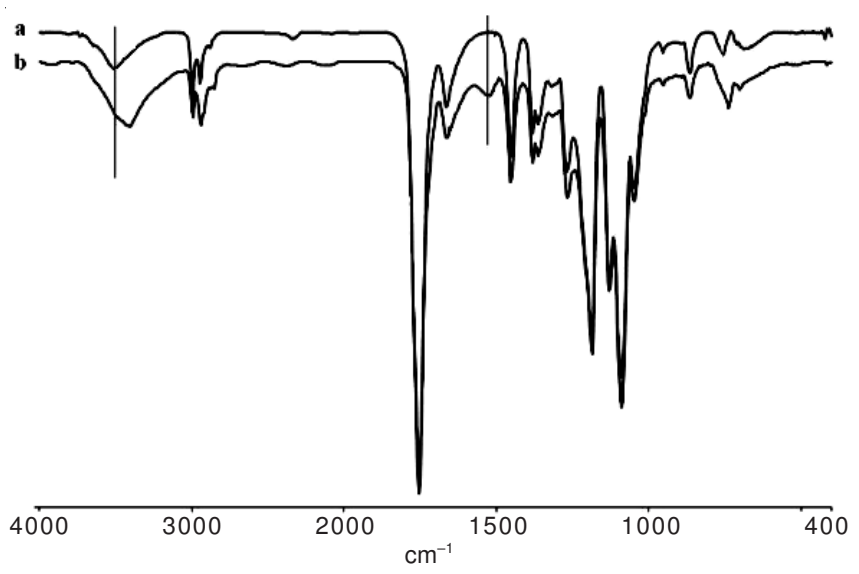

Fig. 1. FT-IR spectra of PDLLA diol (a), BPUU2K1.1 (b)

Mechanical properties of biodegradable poly(urethaneureas): The mechanical properties were determined by dynamic mechanical analysis (DMA) and the storage modulus (Fig. 3i) and $\tan \delta$ (Fig. 3ii) of BPUUs were shown in Fig. 3. It was reported that the $\mathrm{T}_{\mathrm{g}}$ of polymer could be detected and calculated by tan d of polymer in DMA (Table-1), which would be more precisely than $\mathrm{Tg}$ obtained by differential scanning calorimeter $(\mathrm{DSC})^{16}$. From the results of dynamic mechanical analysis, we could easily observed that before the $\mathrm{T}_{\mathrm{g}}$ of BPUU, the storage modulus of BPUUs increased from $2523 \mathrm{MPa}$ to $3295 \mathrm{MPa}$ and the $\mathrm{T}_{\mathrm{g}}$ of the BPUUs also increased from $29.35^{\circ} \mathrm{C}$ to 55.63 ${ }^{\circ} \mathrm{C}$ with hard segment raising. These results were attributed to the increase the content of hard segments, which included an abundant of the rigid piperazine rings and rigid urethane/urea
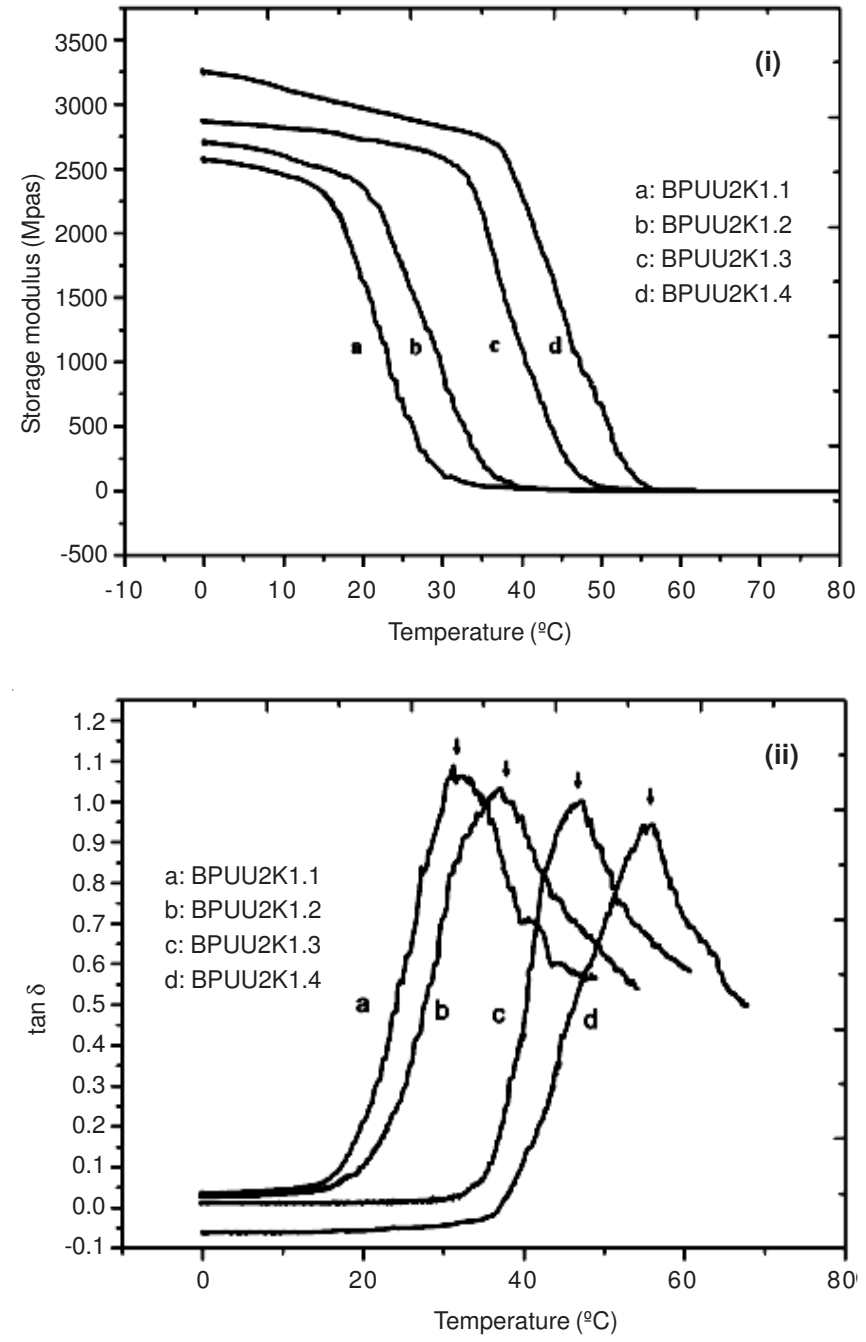

Fig. 3. DMA results of BPUUS: (i) storage modulus; (ii) $\tan \delta$; a: BPUU2K1.1, b: BPUU2K1.2, c: BPUU2K1.3, d: BPUU2K1.4)

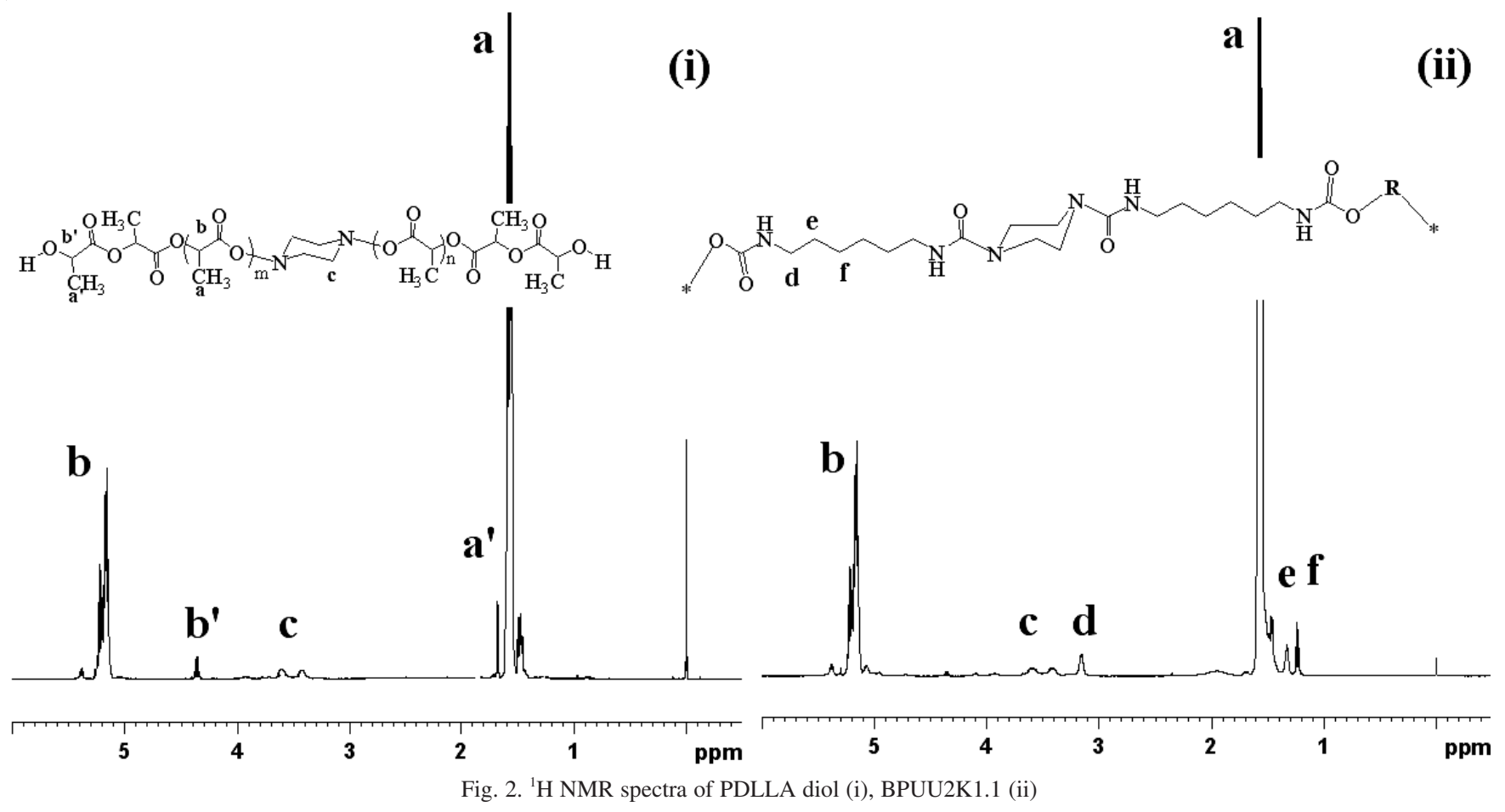



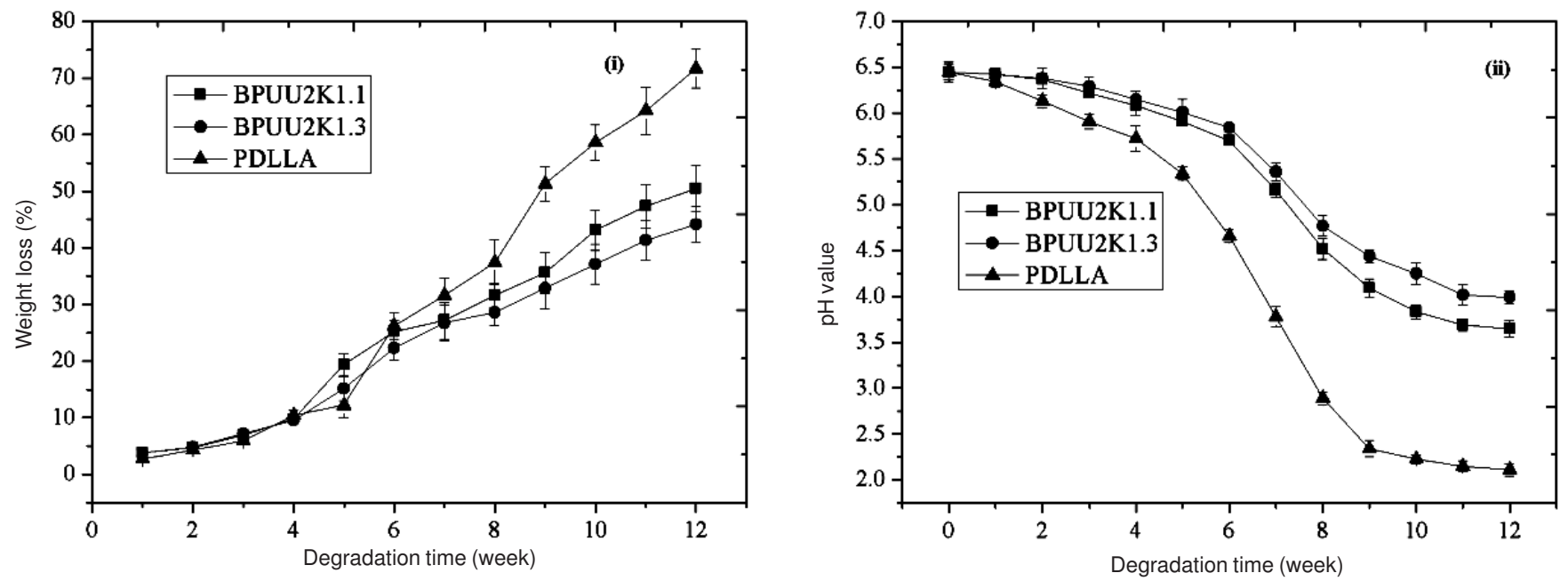

Fig. 4. Degradation of BPUUs and PDLLA within 12 weeks: (i) weight loss in PBS; (ii) pH value in distilled water

groups, which restricted the mobility of soft segments. Besides, hydrogen bonds might also form between the carbonyl groups in PDLLA diol and the -NH- in urethane groups and urea groups, further hindering PDLLA diol chains rotation and increasing $\mathrm{T}_{\mathrm{g}}$ and storage modulus of the polyurethane. Therefore, it could be concluded that increasing the piperazine and hard segments contents could improve the mechanical properties to satisfy the demand of medical implantation.

Biodegradable properties of biodegradable poly(urethane-ureas): The biodegradable property of polymer is the key factor for its application, especially for biomedical engineering. To testify the biodegradation of BPUUs, in vitro degradation tests were carried out at $37{ }^{\circ} \mathrm{C}$, the weight loss ratio (Fig. 4i) of BPUUs in PBS solution and the $\mathrm{pH}$ variation (Fig. 4ii) of BPUUs in double distilled water were monitored once a week for 12 weeks. The weight loss rate of PDLLA was $71.63 \%$ after soaking PBS for 12 weeks, while BPUU2K1.1 and BPUU2K1.3 were $50.44 \%$ and $44.13 \%$, respectively. The tendency of the $\mathrm{pH}$ change of three polymers was the same, but the $\mathrm{pH}$ of BPUUs changed slower than those of PDLLA, which could be attributed to the alkaline groups from breakage of urethane and urea bonds neutralized the acidity, which made the acidity-caused auto-accelerating degradation decreased and the degradation rate decreased. Those results revealed that piperazine played an important role in degradation of BPUUs and could improve the stability of BPUUs in degradation process. Therefore, we will obtain the controlled biodegradation polymers for biomedical application by accommodating the contents of piperazine.

\section{Conclusion}

FTIR spectra and ${ }^{1} \mathrm{H}$ NMR revealed the successful synthesis of novel biodegradable poly(urethane-urea)s based on PDLLA diol, HDI and piperazine. The DMA detections demonstrated that the $\mathrm{T}_{\mathrm{g}}$ values and the mechanical properties BPUUs increased with increasing the hard segments. Mean- while, the piperazine as the chain extender s can improve the biodegradable stability of BPUUs and regulate its degradation rate. Therefore, this biodegradable polyurethane can be designed as potential biomaterials for biomedical fields.

\section{ACKNOWLEDGEMENTS}

Financial supports from the National Natural Science Foundation of China (No. 31200723) and the Development of Strategic Emerging Industries of Shenzhen Basic Research Project (JCYJ20120617122433296) are greatly appreciated.

\section{REFERENCES}

1. J.A. Werkmeister, R. Adhikari, J.F. White, T.A. Tebb, T.P.T. Le, H.C. Taing, R. Mayadunne, P.A. Gunatillake, S.J. Danon and J.A.M. Ramshaw, Acta Biomater., 6, 3471 (2010).

2. J.L. Ryszkowska, M. Auguscik, A. Sheikh and A.R. Boccaccini, Comp. Sci. Technol., 70, 1894 (2010).

3. R. Adhikari, P.A. Gunatillake, I. Griffiths, L. Tatai, M. Wickramaratna, S. Houshyar, T. Moore, R.T.M. Mayadunne, J. Field, M. McGee and T. Carbone, Biomaterials, 29, 3762 (2008).

4. J.N. Zhang, M.Y. Wu, J.J. Yang, Q.Y. Wu and Z.L. Jin, Colloids Surf. A., 337, 200 (2009).

5. B.K. Kim, Exp. Polym. Lett.. 4, 589 (2010)

6. W.S. Wang, Y.L. Guo and J.U. Otaigbe, Polymer, 49, 4393 (2008).

7. P. Woz'niak, M. Bil, J. Ryszkowska, P. Wychowan'ski, E. Wróbel, A. Ratajska, G. Hoser, J. Przybylski, K.J. Kurzydlowski and M. LewandowskaSzumiel, Acta Biomater, 6, 2484 (2010).

8. E. Yilgor and S. Yilgor, Polymer, 42, 953 (2001).

9. Y.L. Wang, M.N. Huang,Y.F. Luo and Y.G. Li, Polym. Degrad. Stab., 95, 549 (2010).

10. Y.L.Wang, C.S. Ruan, J.X. Sun, M.L. Zhang, Y.L. Wu and K. Peng, Polym. Degrad. Stab., 96, 1687 (2011).

11. S.L. Hale and R.A. Kloner, J. Cardiovasc. Pharm. Therap., 11, 249 (2006).

12. B. Astier, L.L. Senas and F. Souliere, Eur. J. Pharmacol., 459, 17 (2003).

13. C.S. Ruan, Y.L. Wang, M.L. Zhang, Y.F. Luo, C.H. Fu, M.N. Huang, J.X. Sun and C.B. Hu Polym. Int., 61, 524 (2012).

14. X.-H. Qu, Q. Wu, J. Liang, X. Qu, S.-G. Wang and G.-Q. Chen, Biomaterials, 26, 6991 (2005).

15. Y.F. Luo, Y.L. Wang, X.F. Niu, C.H. Fu and S.J. Wang, Eur. Polym. J., 43, 3856 (2007).

16. D. Chen and H.G. Zachmann, Polymer, 32, 1612 (1991). 